\title{
Vascularized Ribs in the Treatment of Posterior Instrumentation Infection after Thoracic Vertebrectomy. Case Report
}

\author{
Pedro C. Cavadas ${ }^{1,2}$ Magdalena Baklinska ${ }^{1}$ Daniela Téllez ${ }^{1}$ \\ ${ }^{1}$ Clinica Cavadas, Reconstructive Surgery, Valencia, Spain \\ 2 Departamento de Cirugía, Facultad de Ciencias de la Salud, \\ Universidad Cardenal Herrera-CEU, CEU Universities, C/Santiago \\ Ramón y Cajal, s/n., Valencia, Spain \\ Address for correspondence Pedro C Cavadas, MD, PhD, Clinica \\ Cavadas, Paseo Facultades 1, Valencia 46021, Spain \\ (e-mail: pcavadas@clinicacavadas.es). \\ Indian J Plast Surg 2022;55:107-110.
}

\author{
Abstract \\ Keywords \\ - Vertebrectomy For \\ Bone Metastasis \\ - breast cancer \\ - septic vertebral non- \\ union \\ - vascularized ribs \\ - posterior \\ instrumentation
}

Isolated vertebral mestastases can be successfully treated by surgical removal of the affected vertebral body and stabilization with alloplastic material. The bone union between the upper and lower vertebra is expected, yet a relatively high rate of a nonunion has been reported. In case of infection, bone consolidation is altered, and removal of alloplastic material is recommended, which decreases spinal stability and is a devastating complication.

This case report of delayed infection and exposure of posterior hardware after thoracic vertebrectomy without an interbody osseous union, authors present a successful treatment with vascularized rib flaps through an anterior approach followed by hardware removal.

\section{Introduction}

Vertebrectomy and spinal stabilization is an accepted treatment of isolated vertebral metastases, with favorable results in published series.1,2 Usually performed in a single stage through an anterior, posterior or posterolateral approach, resection of the vertebral body requires reconstruction with an interbody alloplastic cage, bone grafts, and spine stabilization with posterior instrumentation. The bone union between the vertebrae above and below the resected one is the goal, although nonunion rates of $16-50 \%$ have been reported. ${ }^{3,4}$ If the bone union is not achieved, the posterior hardware may fail in the long term due to elastic fatigue, precipitating spinal instability.

Hardware infection after vertebrectomy is a devastating complication, occurring in up to $30 \%$ of the cases in large series. ${ }^{5}$ Hardware removal is usually necessary for infection control, with spinal stability relaying on anterior solid bone union. In the absence of anterior (interbody) bone union, hardware infection is difficult to treat. The authors present a case of delayed infection and exposure of posterior hardware after thoracic vertebrectomy without the interbody bony union, treated with vascularized rib flaps through anterior approach followed by hardware removal. Although vascularized ribs have been described (as a second choice after free fibula) for vascularized bone reconstruction in the spine, ${ }^{6,7}$ to the best of the authors' knowledge, the use of vascularized ribs for staged reconstruction in the setting of infected hardware has not been reported in the English literature.

\section{Case Report}

A 49-year-old woman presented with infected and exposed posterior hardware 10 months after thoracic Th8 published online

February 20, 2022
DOI https://doi.org/ 10.1055/s-0041-1740526. ISSN 0970-0358.

\footnotetext{
(c) 2022. Association of Plastic Surgeons of India. All rights reserved. This is an open access article published by Thieme under the terms of the Creative Commons Attribution-NonDerivative-NonCommercial-License, permitting copying and reproduction so long as the original work is given appropriate credit. Contents may not be used for commercial purposes, or adapted, remixed, transformed or built upon. (https://creativecommons.org/ licenses/by-nc-nd/4.0/) Thieme Medical and Scientific Publishers Pvt. Ltd., A-12, 2nd Floor, Sector 2, Noida-201301 UP, India
} 


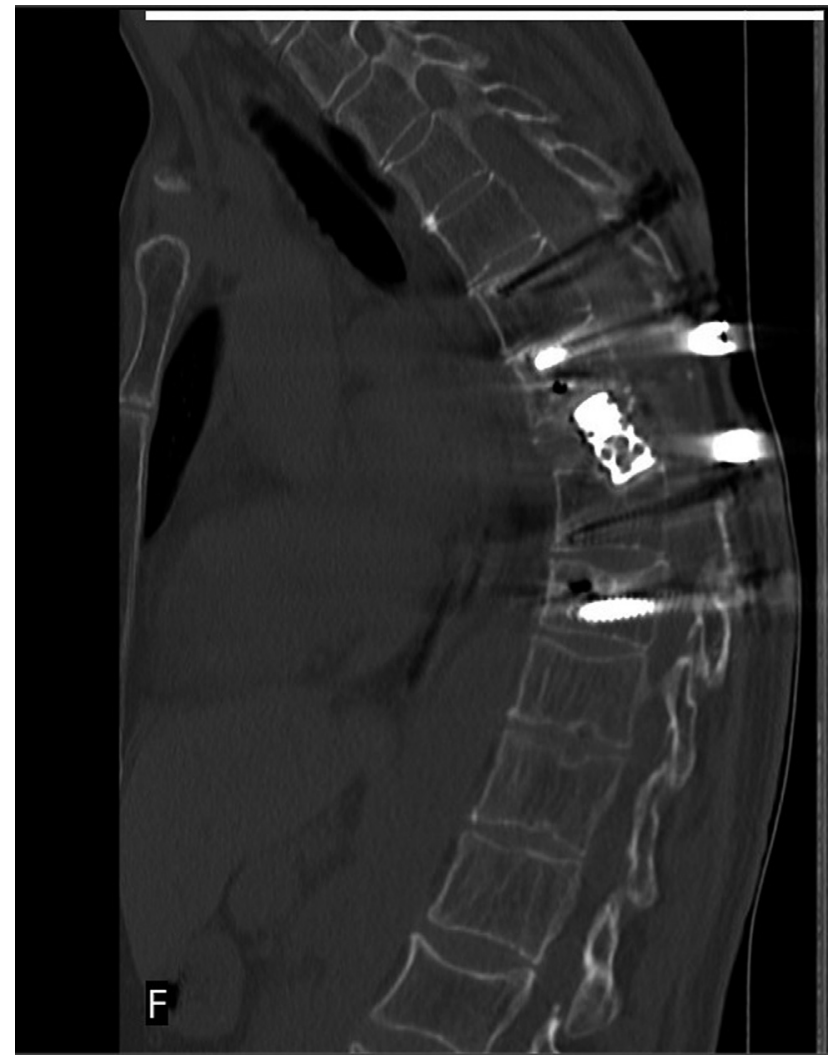

Fig. 1 Sagittal computed tomography (CT) of the spine showing the intervertebral expandable cage without anterior bone union. The posterior hardware was infected, exposed, and unstable.

vertebrectomy for bone metastasis of a breast ductal carcinoma. An expandable interbody cage loaded with autologous iliac bone graft had been used in the previous surgery through a posterior approach, and adjuvant radiation therapy had been given ( $50 \mathrm{~Gy}$ ). There were no signs of bone union between Th7 and Th9 vertebrae (-Fig. 1). Purulent discharge through the back wound, severe pain, and septic signs were present. No pedal pulses were palpable bilaterally. No surgical treatment had been offered to the patient because of the high risk of spinal destabilization should the posterior instrumentation be removed.

The patient was treated with a staged procedure to achieve bone union before hardware removal. Previous radiation therapy discouraged the use of further standard bone grafts and dictated the use of a vascularized bone transfer (VBT). Because of absent pedal pulses, a free fibular flap was not possible, and both iliac crests had been previously harvested as bone grafts at the time of the vertebrectomy.

In the first stage, a VBT was performed through a right lateral thoracotomy. The right thorax was entered between ribs 5th and 6th. There was extensive scarring at the anatomic site of the Th8 body, without draining sinuses or signs of infection of the interbody cage. The scarred mediastinal pleura and periosteum of the anterior and lateral aspect of the vertebral bodies of Th7 and Th9 were resected, and the cortical bone was drilled away to bleeding cancellous bone. The right segmental intercostal vessels were preserved. No attempt was made at exposing or removing the interbody
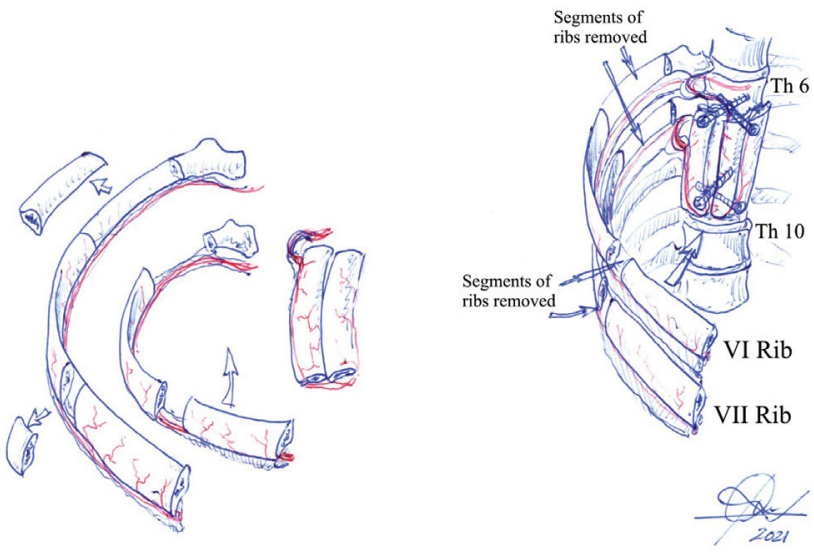

Fig. 2 Drawing of the surgical procedure. Left: the ribs were harvested based on the intercostal bundle. Two segments of bone were removed subperiosteally. The posterior segment allowed pedicle lengthening, and the anterior one allowed a double rib strut to be folded. Right: the 6th and 7th ribs were used in the way depicted to provide four struts of vascularized rib, fixed to the bodies of Th7 and Th8 vertebrae with screws.

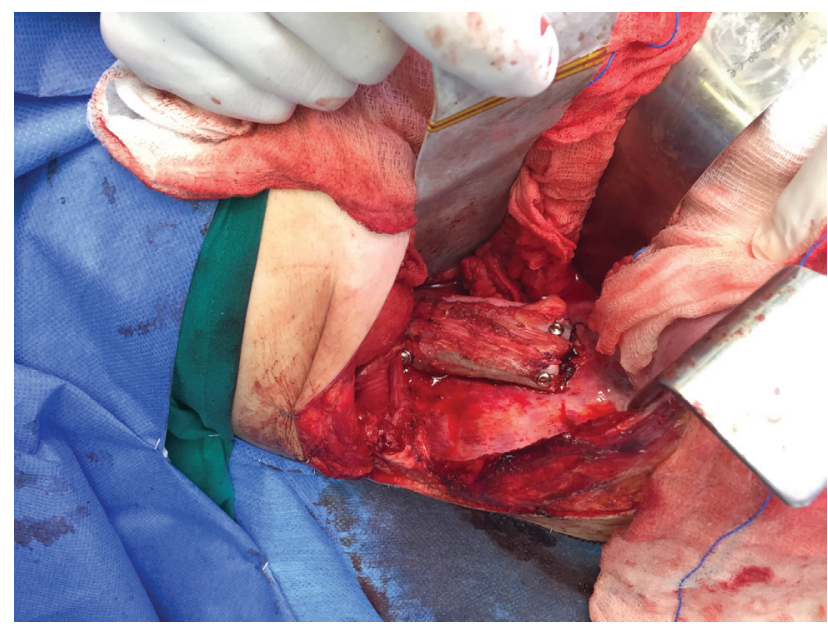

Fig. 3 Intraoperative image of the four segments of vascularized 6th and 7 th ribs inset and fixed with $3.5 \mathrm{~mm}$ screws to the anterolateral aspect of the vertebral bodies Th8 and Th10.

cage. The 6 th and 7 th ribs were harvested vascularized on the intercostal vessels. The two ribs were further divided into two segments each, preserving intercostal pedicles and providing four segments of approximately $7 \mathrm{~cm}$ in length. Enough rib bone was removed posteriorly to allow tensionfree in-setting of the four vascularized rib segments, with maximum contact with the cancellous bone of Th7 and Th9 bodies. Fixation was performed with $3.5-\mathrm{mm}$ screws (-Fig. 2). Special care was taken to avoid compression of the segmental vessels or kinking of the intercostal pedicles. The resultant full-thickness defect of the chest wall was reconstructed with a sandwich of polymethyl methacrylate (with vancomycin and gentamycin) and a polypropylene mesh. A pleural drain was placed, and the thoracotomy wound was closed.

The patient was given oral rifampicin and cotrimoxazole to suppress the dorsal infection. Three months later, after bone healing was evident between Th7 and Th9 vertebral 


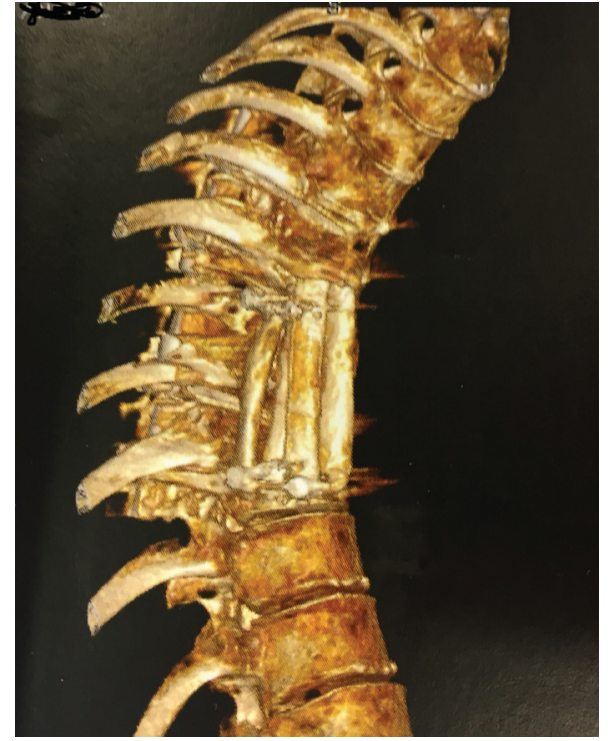

Fig. 4 3D computed tomography (CT) image of the healed rib segments.

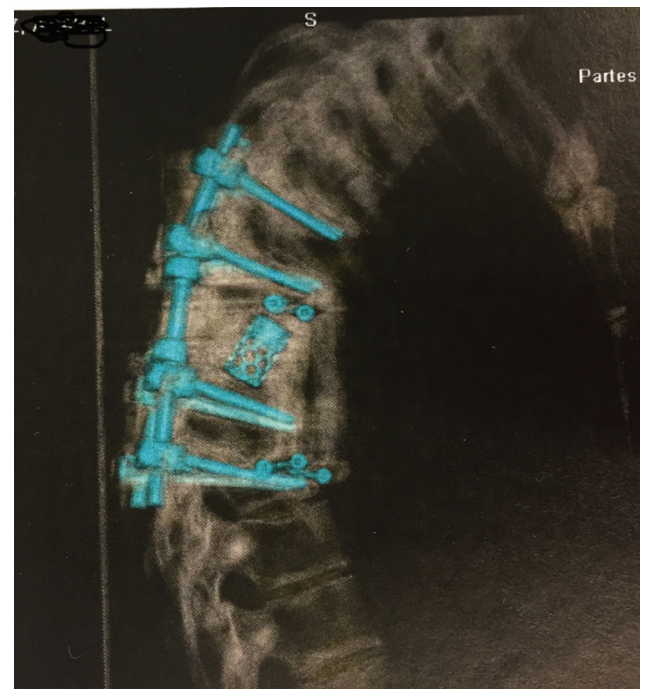

Fig. 5 3D computed tomography (CT) semitransparent image showing the bone reconstruction before posterior instrumentation removal.

bodies and the rib struts on the CT scan (-Figs. 3-8), the posterior instrumentation was removed, and the dorsal wound was primarily closed. The patient wore a removable corset for added safety for 1 month and then was allowed normal movement. No further kyphotic deformity or medullary compression symptoms developed, and C-reactive protein (CRP) levels normalized soon after hardware removal. One year after the second procedure, the patient is symptom-free with no evidence of further neoplastic progression.

\section{Discussion}

With improvements in surgical technique and oncological treatment, resection of isolated vertebral metastases may be

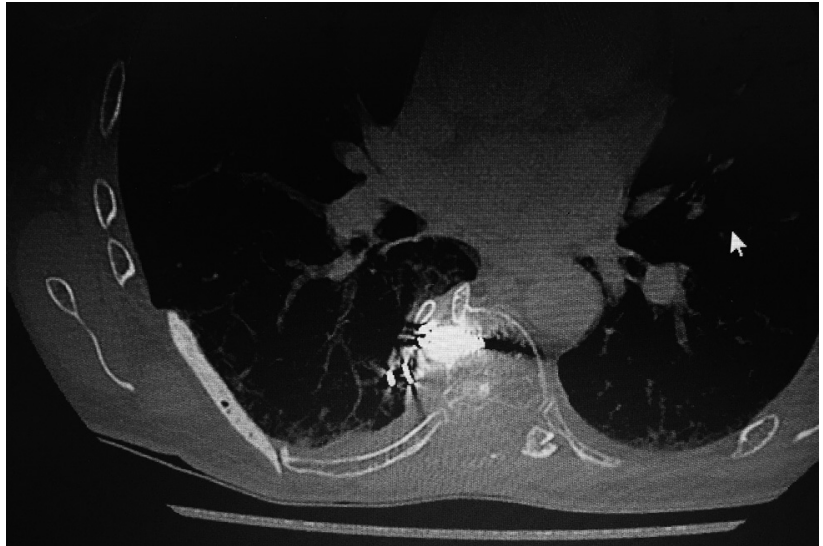

Fig. 6 Computed tomography (CT) scan after posterior hardware removal, showing bone healing at Th8 level (with metallic artifacts from the crews).

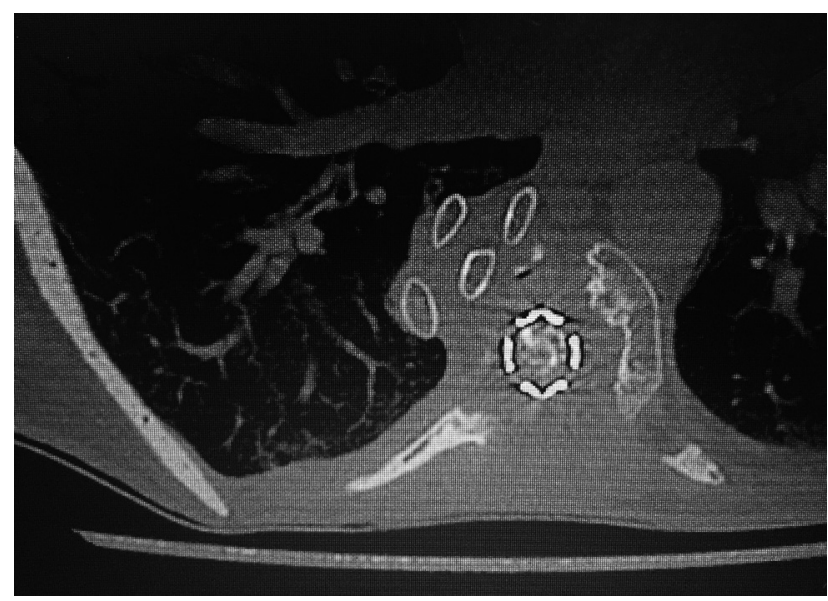

Fig. 7 Computed tomography (CT) scan after posterior hardware removal, showing rib bone cross-section at Th9 level. The sandwich polymethyl methacrylate (PMMA) mesh is visible at the donor chest wall.

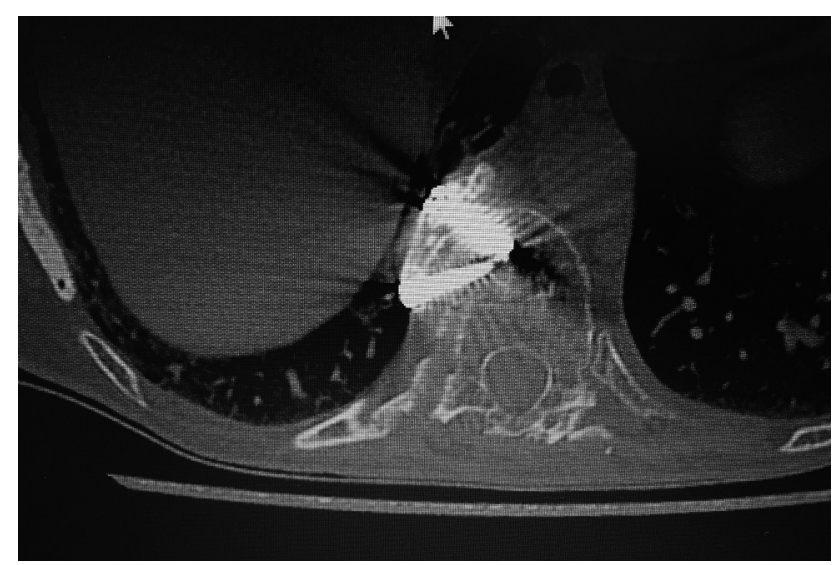

Fig. 8 Computed tomography (CT) scan after posterior hardware removal, showing bone healing at Th10 level (with metallic artifacts from the crews).

beneficial to the patientś survival and quality of life., ${ }^{1,2}$ Achievement of interbody bone fusion is paramount for mid- and long-term spinal stability ${ }^{3,4}$. The advantage of 
vascularized bone over conventional bone grafts has been well-established in unfavorable situations (long defects, irradiated or previously infected wounds) and also in spinal reconstruction. ${ }^{5-9}$ Despite ample experimental and clinical evidence of this superiority, the use of vascularized bone for primary reconstruction after en bloc vertebral resection is anecdotal. Most of the time, particularly bone grafts, either autograft or mixed auto and allograft, are used with interbody cages, with few series of free fibula VBT in this subset of patients. In a recent series, the primary use of free fibula VBT was predictive of the bone union after en bloc vertebrectomy. ${ }^{10}$ The added complexity of a free flap to an already lengthy and complex procedure probably explains the more frequent use of standard bone grafts, even at the expense of accepting an increased risk of nonunion. ${ }^{10}$

Deep infection of the posterior instrumentation after vertebrectomy is a serious complication that often requires hardware removal. ${ }^{11}$ If the bone union has not been achieved (a not infrequent situation), hardware removal severely compromises spinal stability and can precipitate a disastrous spinal cord compression. In the presence of active infection, interbody bone fusion can only be achieved through a clean, alternative approach. Hardware removal can safely be performed in the second stage. In the case presented herein, the vertebrectomy, interbody cage placement, and instrumentation was performed through a posterior approach. This was a fortunate situation since it allowed an anterior approach through a right lateral thoracotomy to achieve bone union in a clean environment. Segmental branches of the thoracic artery had not been ligated, although the vessels were encased in a thick scar. This fact and the unavailability of more common VBT (fibula or iliac crest) made the senior author decide on a pedicle double rib flap. Vascularized ribs have been described in spinal reconstruction, mainly as a second choice, the first choice being free fibular flaps. ${ }^{5,12}$ Pedicled ribs are faster and easier to execute compared with free flaps at the expense of a weaker construct. The ribs have a thinner bony structure compared with the fibula, so in this case, four segments were used to increase the fusion mass. In a normal adult, the cross-section of the 6 th and 7 th ribs is approximately $0.5 \mathrm{~cm}^{2}$, so a $2 \mathrm{~cm}^{2}$-thick construct of vascularized bone was fixed to the anterior surface of the vertebral bodies Th7 and Th9 with screws, and the fusion mass proved to be enough to allow hardware removal without spinal destabilization (-Fig. 6).

Alloplastic reconstruction of the resultant chest wall defect had a theoretical risk of infection, although the remote location from the infected dorsal wound and the use of antibioticloaded cement allowed an uneventful postoperative.

The use of VBT, either free fibular or iliac crest or pedicled vascularized ribs placed through a clean alternative ap- proach, should be considered as a means of achieving bone fusion before infected posterior instrumentation can be safely removed in patients with postvertebrectomy septic complications.

\section{Informed Consent}

Written, informed consent was obtained from the patient, including her consent to publish pictures.

\section{Funding}

Our work, presented in this paper, was not subject to any external funding or financial involvement or support

Conflict of Interest

The authors have no conflict of interests.

\section{References}

1 Joubert C, Adetchessi T, Peltier E, et al. Corpectomy and vertebral body reconstruction with expandable cage placement and osteosynthesis via the single stage posterior approach: a retrospective series of 34 patients with thoracic and lumbar spine vertebral body tumors. World Neurosurg 2015;84(05):1412-1422

2 Akeyson EW, McCutcheon IE. Single-stage posterior vertebrectomy and replacement combined with posterior instrumentation for spinal metastasis. J Neurosurg 1996;85(02):211-220

3 Emery SE, Hughes SS, Junglas WA, Herrington SJ, Pathria MN. The fate of anterior vertebral bone grafts in patients irradiated for neoplasm. Clin Orthop Relat Res 1994;(300):207-212

4 Lonstein JE, Winter RB. Long multiple struts for severe kyphosis. Clin Orthop Relat Res 2002;(394):130-138

5 Shin AY, Dekutoski MB. The role of vascularized bone grafts in spine surgery. Orthop Clin North Am 2007;38(01):61-72, vi

6 Arata MA, Wood MB, Cooney WP III. Revascularized segmental diaphyseal bone transfers in the canine. An analysis of viability. J Reconstr Microsurg 1984;1(01):11-19

7 Cutting CB, McCarthy JG. Comparison of residual osseous mass between vascularized and nonvascularized onlay bone transfers. Plast Reconstr Surg 1983;72(05):672-675

8 Shaffer JW, Field GA, Goldberg VM, Davy DT. Fate of vascularized and nonvascularized autografts. Clin Orthop Relat Res 1985; (197):32-43

9 Shaffer JW, Field GA. Rib transposition vascularized bone grafts. Hemodynamic assessment of donor rib graft and recipient vertebral body. Spine 1984;9(05):448-449

10 Mericli AF, Boukovalas S, Rhines LD, Adelman DM, Hanasono MM, Chang EI. Free fibula flap for restoration of spinal stability after oncologic vertebrectomy is predictive of bony union. Plast Reconstr Surg 2020;145(01):219-229

11 Araujo AO, Narazaki DK, Teixeira WGJ, et al. En bloc vertebrectomy for the treatment of spinal lesions. Five years of experience in a single institution: a case series. Clinics (São Paulo) 2018;73: e95

12 Wilden JA, Moran SL, Dekutoski MB, Bishop AT, Shin AY. Results of vascularized rib grafts in complex spinal reconstruction. J Bone Joint Surg Am 2006;88(04):832-839 